\title{
Petahertz optical drive with wide-bandgap semiconductor
}

\author{
Hiroki Mashiko ${ }^{1 \star}$, Katsuya Oguri ${ }^{1}$, Tomohiko Yamaguchi ${ }^{1,2}$, Akira Suda ${ }^{2}$ and Hideki Gotoh ${ }^{1}$
}

\begin{abstract}
High-speed photonic and electronic devices at present rely on radiofrequency electric fields to control the physical properties of a semiconductor ${ }^{1}$, which limits their operating speed to terahertz frequencies $\left(10^{12} \mathrm{~Hz}\right.$; ref. 2). Using the electric field from intense light pulses, however, could extend the operating frequency into the petahertz regime $\left(10^{15} \mathrm{~Hz}\right.$; ref. 3). Here we demonstrate optical driving at a petahertz frequency in the wide-bandgap semiconductor gallium nitride. Few-cycle near-infrared pulses are shown to induce electric interband polarization though a multiphoton process. Dipole oscillations with a periodicity of 860 as are revealed in the gallium nitride electron and hole system by using the quantum interference between the two transitions from the valence and conduction band states, which are probed by an extremely short isolated attosecond pulse with a coherent broadband spectrum. In principle, this shows that the conductivity of the semiconductor can be manipulated on attosecond timescales, which corresponds to instantaneous light-induced switching from insulator to conductor. The resultant dipole frequency reaches $1.16 \mathrm{PHz}$, showing the potential for future highspeed signal processing technologies based on wide-bandgap semiconductors.
\end{abstract}

The growing demand for unprecedented speeds and capacities in the advanced information society has been creating challenges in signal processing technology based on modern semiconductor photonics and electronics. High-speed operation depends on ultrafast control of physical properties with radiofrequency (RF) electric fields ${ }^{1}$. This principle is common to semiconductor applications ranging from the field-effect transistor, which is one of the building blocks for high-clock-rate logic operation in digital electronics, to the electro-absorption modulator for high-bitrate transport in optical communications ${ }^{2,4-7}$. In such high-speed semiconductor devices, the maximum operational frequency is in the terahertz $\left(10^{12} \mathrm{~Hz}\right)$ regime ${ }^{2}$, which is limited by the response time of band energy modulation with RF electric fields and by the temporal characteristics of carrier transit time (mobility) and carrier lifetime (recombination $)^{7}$.

To extend the operational frequency into the petahertz $\left(10^{15} \mathrm{~Hz}\right)$ regime, lightwave field control has potential ${ }^{3}$. An isolated attosecond pulse (IAP) source ${ }^{8-11}$ has the ability to detect such ultrafast electron dynamics, as experimentally demonstrated in atomic $^{12-15}$, molecular ${ }^{16}$, and solid ${ }^{17,18}$ systems. Our approach uses the attosecond dynamics of electronic interband polarization in the wide-bandgap semiconductor gallium nitride $(\mathrm{GaN})$ induced by a few-cycle near-infrared (NIR) pulse. The extremely short IAP with a coherent broadband spectrum monitors the quantum interference between the valence band (VB) and conduction band
(CB) states, which reveals an electronic dipole oscillation with attosecond periodicity.

GaN semiconductor, with its high breakdown voltage, high heat capacity, and high thermal conductivity, is a promising material ${ }^{19}$ because it supports interband polarization efficiently without high-intensity light injection and serious optical damage. In this experiment, we used epitaxially grown bulk GaN with the wurtzite (hexagonal) structure [0001] as a target. Figure 1a shows a schematic view of the experimental set-up for transient absorption spectroscopy with GaN using the IAP and NIR pulse (for details of the system, see Supplementary Section 1). Figure $1 \mathrm{~b}$ shows the energy level diagram of intrinsic $\mathrm{GaN}$ (ref. 20). The Fermi level $E_{\mathrm{F}}$ and the work function $\phi$ are 1.7 and $4.5 \mathrm{eV}$, respectively. GaN allows a direct optical transition at the $\Gamma$ point on wavevector $K$ (ref. 21). The NIR pulse induces ultrafast electronic interband polarization through a multiphoton process in the semiconductor material ${ }^{22}$. Simultaneously, the IAP (centre photon energy of $20.5 \mathrm{eV}$ ) allows the excitation of electrons from both the VB and CB. Here we define the high-energy levels of the $\mathrm{CB}$ with the IAP excitation as the continuum state. Figure 1c shows the measured absorption spectrum in the energy region from 1 to $6 \mathrm{eV}$. The bandgap energy $E_{\mathrm{g}}$ is $3.35 \mathrm{eV}$ (blue filled circles) at room temperature. An impurity state between the VB and CB does not exist in GaN. The NIR pulse (red shaded area) has a centre photon energy of $1.6 \mathrm{eV}$. Therefore, the interband transition from the $\mathrm{VB}$ to the $\mathrm{CB}$ requires a process with at least three photons in $\mathrm{GaN}^{23}$.

In this experiment, a few-cycle pulse (7-fs duration) from a Ti:sapphire laser was used for high-harmonic generation and as a NIR pump pulse for the transient absorption spectroscopy. The IAP is generated by means of the double optical gating (DOG) technique using argon gas ${ }^{24}$. The IAP has a duration of 660 as (for details of the IAP temporal characterization, see Methods and Supplementary Section 2). The collinearly propagating IAP and NIR pulse are focused onto stand-alone 102-nm-thick GaN without a substrate. The thin GaN sample is manufactured from millimetre-thick bulk GaN by a combination of mechanical polishing and ion beam milling. In this pump-probe system, the root mean square timing jitter is 23 as. After passing through the GaN, the transmitted IAP is sent to a vacuum ultraviolet (VUV) spectrometer. The spectrometer resolution is $180 \mathrm{meV}$ at a photon energy of $20.5 \mathrm{eV}$.

Figure 2a shows the measured attosecond transient absorption trace. The trace shows the deviation of optical density $(\Delta \mathrm{OD})$ with and without the NIR pulse as a function of time delay (for the definition of $\triangle O D$, see Methods and Supplementary Section 3). The GaN is irradiated by a NIR pulse with an intensity of approximately $1 \times 10^{10} \mathrm{~W} \mathrm{~cm}^{-2}$. The transient absorption trace exhibits characteristic temporal modulation throughout the

${ }^{1}$ NTT Basic Research Laboratories, 3-1 Morinosato Wakamiya, Atsugi, Kanagawa 243-0198, Japan. ${ }^{2}$ Tokyo University of Science, 2641 Yamazaki, Noda-shi, Chiba-ken 278-8510, Japan. *e-mail: mashiko.hiroki@lab.ntt.co.jp 


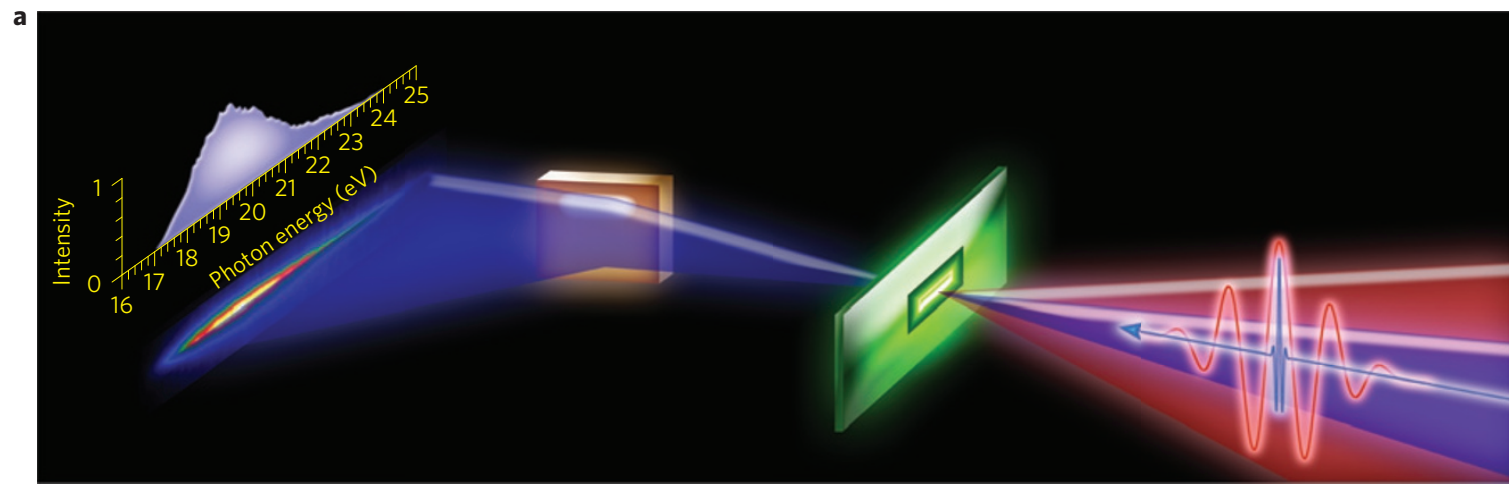

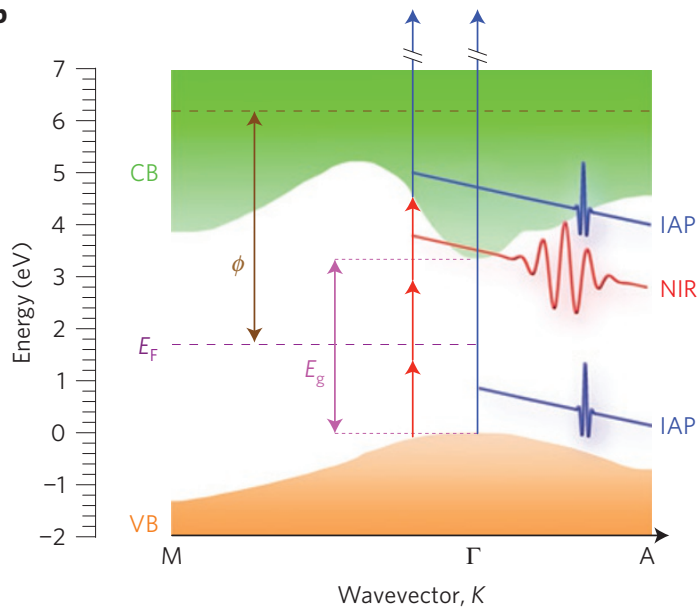

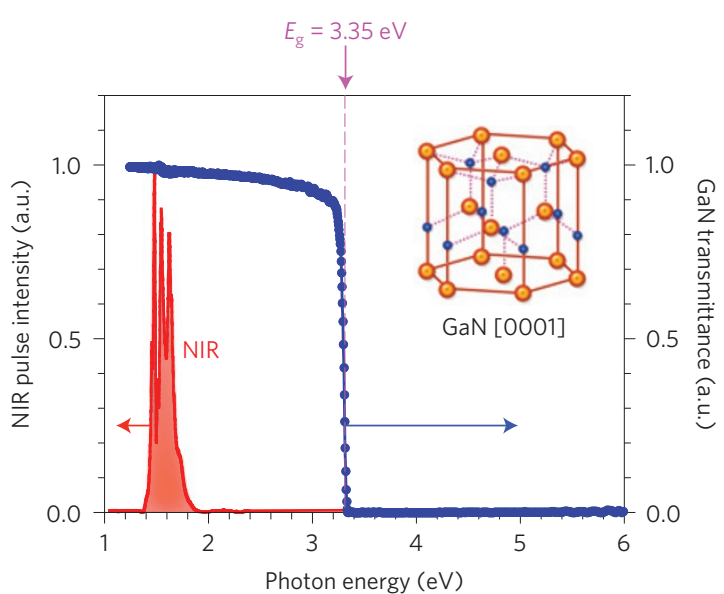

Figure 1 | Experimental set-up and energy level diagram of GaN. a, Schematic view of the experimental set-up for transient absorption spectroscopy with the 102-nm-thick GaN using the IAP (660-as duration) and NIR pulse (7-fs duration). The transmitted IAP is detected by the VUV spectrometer. b, Energy level diagram of wurtzite structure GaN [0001]. Orange and green shaded areas are VB and CB structures at the $M, \Gamma$, and $A$ points in wavevector $K$, respectively. The Fermi level $E_{\mathrm{F}}$ and work function $\phi$ are $1.7 \mathrm{eV}$ (purple dashed line) and $4.5 \mathrm{eV}$ (brown arrow), respectively ${ }^{20}$. Red and blue arrows show the NIR pulse and IAP, respectively. The pink arrow shows the bandgap $E_{g}$. c, Measured transmittance (blue filled circles) and spectrum of the NIR pulse (red solid line). The bandgap is $3.35 \mathrm{eV}$ (pink dotted line) at room temperature.

a

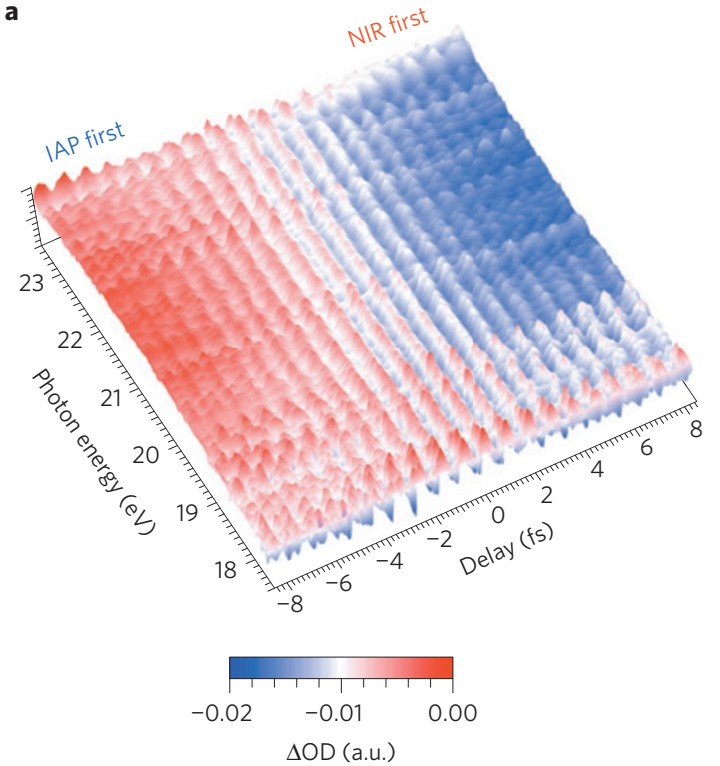

b

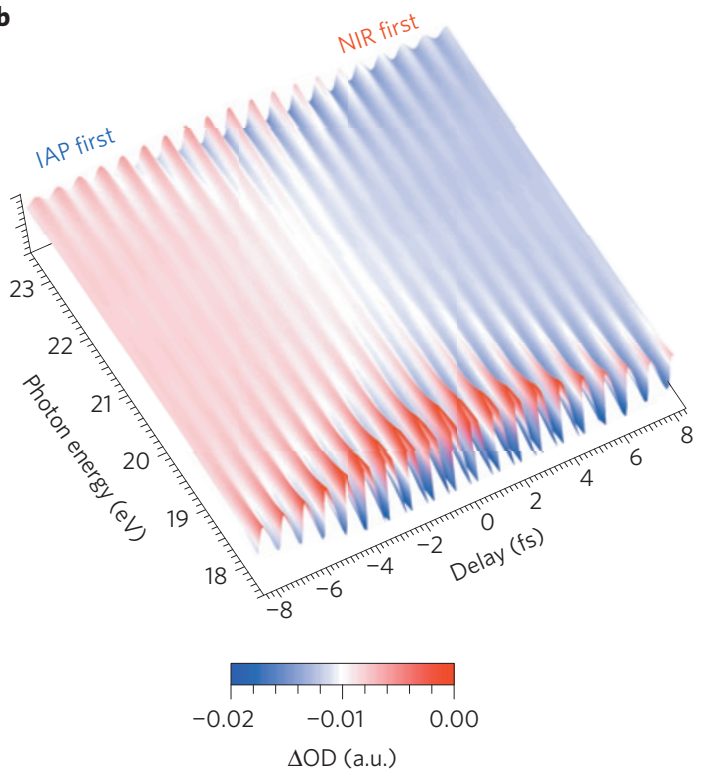

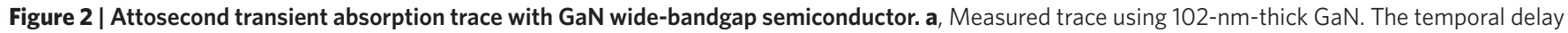
between the IAP and NIR pulse is stepped by 100 as in the measurement. The trace shows the deviation of optical density ( $\Delta$ OD) with and without the NIR pulse as a function of the temporal delay. The definition of $\Delta O D$ is given in the Methods and Supplementary Section 3 . The trace is averaged over 30 measurements. b. Trace of the interband polarization simulated using a multilevel optical Bloch equation ${ }^{26-28}$. 


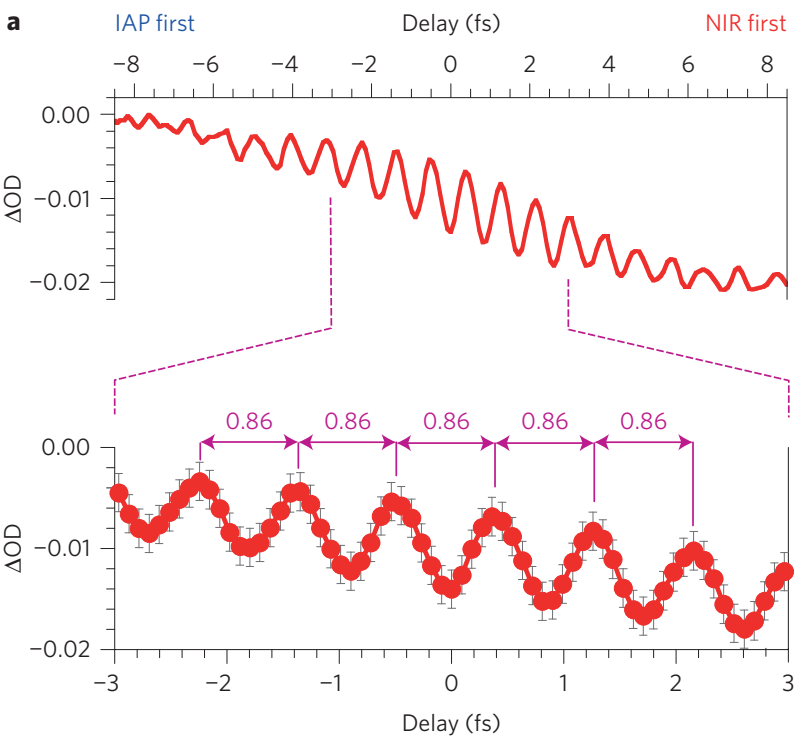

b

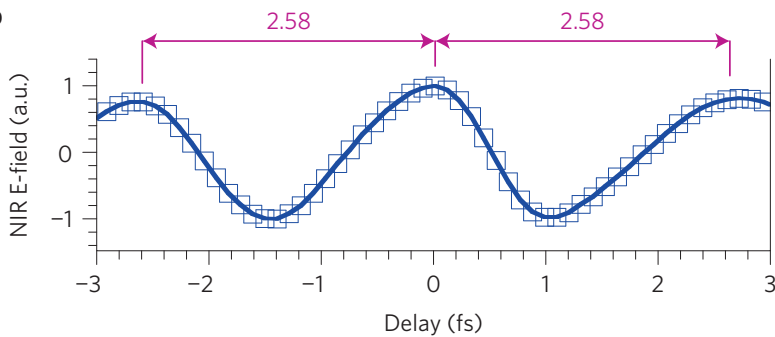

Figure $\mathbf{3}$ | Line profile structures of dipole oscillation. $\mathbf{a}$, The upper graph shows the integrated line profile structure (red solid line) for the 17.5-23.5 eV photon energy region in Fig. 2a. The lower graph shows the line profile structure (red filled circles) in the shorter delay region, from which one can determine that the oscillation periodicity is 860 as (pink arrows). Error bars represent the standard deviation over 30 measurements. b. Electric field of the NIR pulse (blue open squares) reconstructed by the FROG-CRAB method ${ }^{29}$ using krypton atoms. The 2.58-fs duration (pink arrows) corresponds to the full-cycle periodicity of the NIR pulse.

entire energy region, indicating sub-femtosecond electric dipole oscillation in the GaN. When the IAP or NIR pulse couple to another discrete electronic state, the transition opens a new discrete absorption channel ${ }^{15,25}$. However, because the temporal modulation is distributed throughout the entire energy region, as shown in our results, the continuum state transition dominates the absorption process. To develop a better understanding of this observation, we numerically simulated the interband polarization using a multilevel optical Bloch equation ${ }^{26-28}$. The result is shown in Fig. 2b. In this simulation, for an intuitive understanding, we modelled the GaN electronic system as a two-level system in which the ground and excited states mimic the $\mathrm{VB}$ and $\mathrm{CB}$, respectively. Our formulation is based on previous simulations ${ }^{26-28}$, and we extend the previous two-level formalism to a three-level system, taking free continuum states into account (for the calculation model and conditions, see Supplementary Section 4). The coherent broadband IAP excites the superposition state of electrons in the $\mathrm{VB}$ and $\mathrm{CB}$ to the continuum level. The induced coherence of the superposition state leads to quantum interference, which results in a temporal modulation of the IAP absorption spectrum in the entire photon energy region from 17.5 to $23.5 \mathrm{eV}$. The calculated absorption trace reproduces the experimental observation.

Figure 3 shows the line profile structures. Figure 3a (top) shows the integrated line profile (red solid line) for the $17.5-23.5 \mathrm{eV}$ photon energy region in Fig. 2a. The base absorption reduces slightly

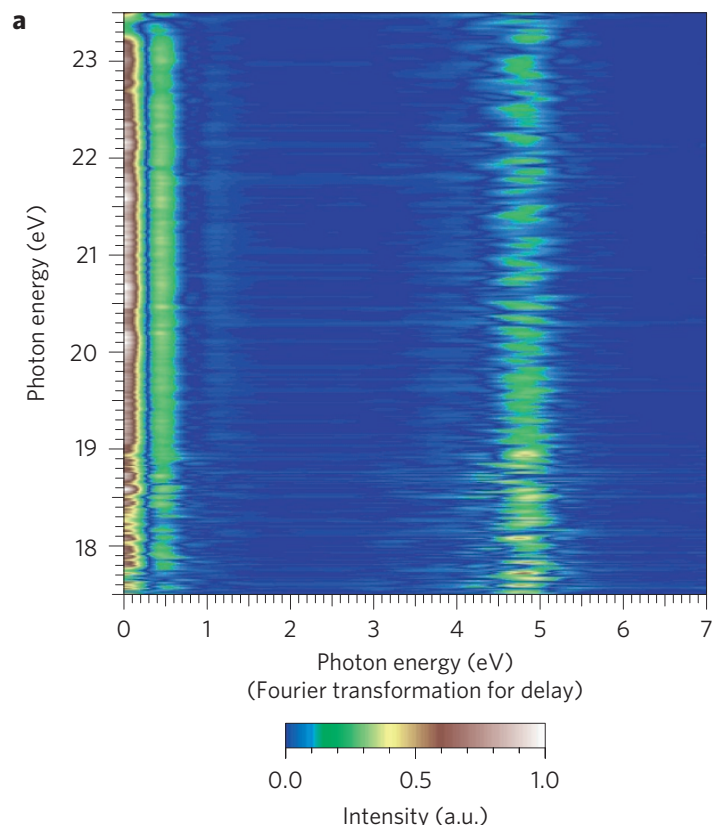

b

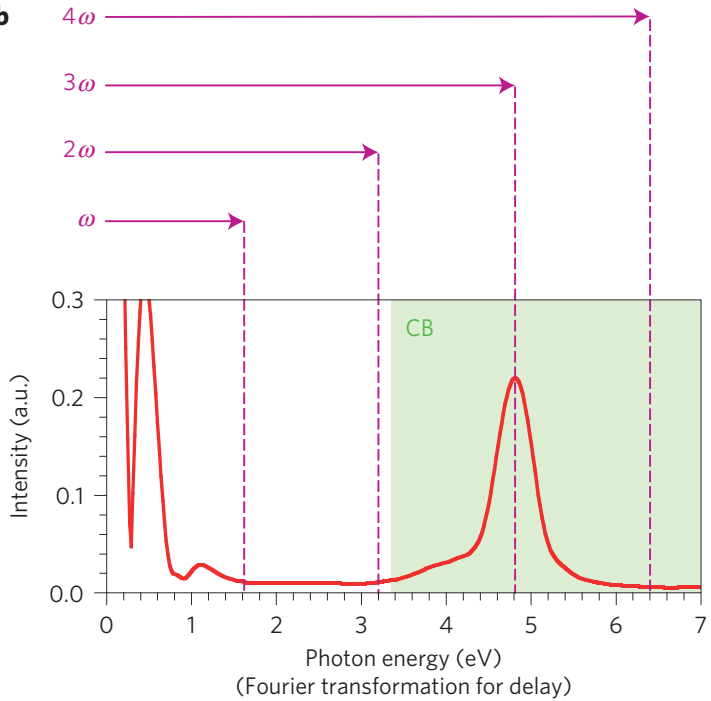

Figure 4 | Photon energy component with Fourier analysis. a, Spectral distribution with Fourier transformation for the temporal delay axis in Fig. 2a. b, Line profile structure (red solid line) integrated for the $17.5-23.5 \mathrm{eV}$ photon energy region in $\mathbf{a}$. The green shaded area shows the CB state. The pink arrows correspond to the $\omega, 2 \omega, 3 \omega$, and $4 \omega$ angular frequency components of the NIR pulse.

as the temporal delay between the IAP and NIR pulse advances. Because the NIR pulse excites electrons from the VB to the $\mathrm{CB}$, the IAP probes the absorption decay (approximately 5\% increase in transmittance). The decay time is estimated to be approximately $7 \mathrm{fs}$, which is almost the same as the duration of the NIR pulse, thus indicating that the coherence time of the induced polarization is comparable to the NIR pulse duration. During the decay, a fine oscillation structure is observed (maximum increment of $2.5 \%$ transmittance in the oscillatory part). The oscillation periodicity is 860 as, as shown in the expanded section in the lower part of Fig. 3a (red solid line with filled circles). The most important feature is that the frequency of the temporal modulation is $1.16 \mathrm{PHz}$. The observed frequency on the attosecond timescale breaks the $1-\mathrm{PHz}$ barrier in electron motion in solid-state materials and is the highest frequency ever reported. For comparison with the oscillation, we 
also temporally characterize the NIR pulse's electric field (in Fig. 3b), which is reconstructed from the frequency-resolved optical gating for complete reconstruction of attosecond bursts (FROG-CRAB) method $^{29}$ based on the attosecond streak effect ${ }^{30}$ using krypton atoms. The measured periodicity is $2.58 \mathrm{fs}$, which corresponds to the full-cycle periodicity of the NIR pulse. Thus, the temporal delay is calibrated well in this system.

Figure $4 \mathrm{a}$ shows the spectral distribution with Fourier transformation of the temporal delay axis in Fig. 2a. The result indicates that the dipole oscillation obviously has an energy component of $4.8 \mathrm{eV}$. Figure $4 \mathrm{~b}$ is the line profile structure corresponding to the integrated $17.5-23.5 \mathrm{eV}$ photon energy region in Fig. 4a. The green shaded area shows the $\mathrm{CB}$ state. The pink arrows correspond to the $\omega, 2 \omega, 3 \omega$, and $4 \omega$ angular frequency components of the NIR pulse. The result indicates that the $3 \omega$ component dominates the temporal modulation. The energy value needs to exceed the barrier of the bandgap of $3.35 \mathrm{eV}$. Thus, the $\omega$ and $2 \omega$ components with lower energy are hardly detected. This observation supports the assumption that the third-order interband polarization is dominant because of the first resonance excitation condition. The temporal variation of the relative phase $\varphi(t)$ between the $\mathrm{VB}$ and $\mathrm{CB}$ is expressed as $\varphi(t)=\Delta E / \hbar \equiv 2 \pi / T$ (ref. 12), where $\hbar$ is Planck's constant divided by $2 \pi$. Here, $\Delta E$ is the transition energy of $4.8 \mathrm{eV}$ corresponding to the $3 \omega$ component of the NIR pulse $(1.6 \mathrm{eV}$ at $\omega)$. The value $\Delta E=4.8 \mathrm{eV}$ implies that the dipole oscillation period $T$ is 860 as. In principle, the ultrafast electron motion observed with the wide-bandgap semiconductor indicates manipulation of the conductivity on the attosecond timescale.

In previous reports, observations of third harmonic generation with spectral and interferometric measurements confirmed that third-order nonlinearity can be induced by interactions between a few-cycle NIR pulse and semiconductors such as $\mathrm{GaAs}^{26}, \mathrm{ZnO}^{27}$, and $\mathrm{TiO}_{2}{ }^{22}$. Because third harmonic generation corresponds to electromagnetic radiation originating from the third-order nonlinear polarization, these studies indirectly suggested electronic dynamics occurring on a sub-optical-cycle timescale. In contrast to these indirect observations, our attosecond transient absorption spectroscopy provides the direct time-domain observation of electronic dipole oscillation resulting in third-order nonlinear polarization. In addition, it is likely that the resonance excitation and the sensitive detection system enable us to detect the third-order nonlinearity with a oneor two-order lower intensity of the NIR pulse $\left(1 \times 10^{10} \mathrm{~W} \mathrm{~cm}^{-2}\right)$ compared to previous reports $\left(10^{11} \sim 10^{12} \mathrm{~W} \mathrm{~cm}^{-2}\right)$. This ultrafast property revealed by the direct time-domain observation will provide an ultrafast manipulation technology for dipole oscillation. The benefit is directly linked to controlling absorption, reflection, refractive indices, photocurrent, photoemission and diffraction, which are important for implementing photonic and electronic devices with unprecedented speed in the future.

In other solid-state experiments using the IAP, Zener tunnelling of electrons in an insulator (quartz) and a step-wise population increase in a semiconductor (silicon) have been reported, both of which were monitored by an inner-shell transition ${ }^{19,20}$. Zener tunnelling is an extreme highly nonlinear effect induced by an intense lightwave field (peak intensity higher than $10^{12} \mathrm{~W} \mathrm{~cm}^{-2}$ ). Because the phenomenon depends on the peak electric field of the NIR pulse, the driving frequency corresponds to a half-cycle period up to $800 \mathrm{THz}$. Here, the advantage of the interband transition using a multiphoton process with the NIR pulse in wide-bandgap $\mathrm{GaN}$ is that a driving frequency higher than $1 \mathrm{PHz}$ can be achieved. In addition, a lightwave field whose intensity is two orders of magnitude lower can be used, thus avoiding serious optical damage to the target material. This result also suggests that when an ultraviolet laser is directly used for the pump field instead of the present NIR pulse, the transition efficiency would be increased and the intensity of the lightwave field could be further reduced.
The demonstrated petahertz optical drive using wide-bandgap semiconductor has the potential for use in constructing high-clockrate logic operation systems in digital photonics and electronics, because the ultrafast electron dynamics in the attosecond time scale activates the semiconductor device with instantaneous switching from insulator to conductor and vice versa. This study shows the potential of future petahertz signal processing technology based on ordinary wide-bandgap semiconductor devices.

\section{Methods}

Methods and any associated references are available in the online version of the paper.

Received 13 November 2015; accepted 1 March 2016; published online 11 April 2016

\section{References}

1. Neamen, D. A. Semiconductor Physics and Devices: Basic Principles (McGraw-Hill, 2011).

2. Mei, X. et al. First demonstration of amplification at $1 \mathrm{THz}$ using $25-\mathrm{nm} \mathrm{InP}$ high electron mobility transistor process. IEEE Electron Device Lett. 36, 327-329 (2015)

3. Krausz, F. \& Stockman, M. I. Attosecond metrology: from electron capture to future signal processing. Nature Photon. 8, 205-213 (2014).

4. Schwierz, F. \& Liou, J. RF transistors: recent developments and roadmap toward terahertz applications. Solid-State Electron. 51, 1079-1091 (2007).

5. Liu, J. et al. Waveguide-integrated, ultralow-energy GeSi electro-absorption modulators. Nature Photon. 2, 433-437 (2008)

6. Reed, G. T., Mashanovich, G., Gardes, F. Y. \& Thomson, D. J. Silicon optical modulator. Nature Photon. 4, 518-526 (2010)

7. Sze, S. M. Physics of Semiconductor Devices (John Wiley, 1981).

8. Goulielmakis, E. et al. Single-cycle nonlinear optics. Science 320, 1614-1617 (2008).

9. Sansone, G. et al. Isolated single-cycle attosecond pulses. Science 314, 443-446 (2006)

10. Mashiko, H. et al. Extreme ultraviolet supercontinua supporting pulse durations of less than one atomic unit of time. Opt. Lett. 34, 3337-3339 (2009)

11. Takahashi, E. J., Lan, P., Mücke, O. D., Nabekawa, Y. \& Midorikawa, K. Attosecond nonlinear optics using gigawatt-scale isolated attosecond pulses. Nature Commun. 4, 2691 (2013).

12. Goulielmakis, E. et al. Real-time observation of valence electron motion. Nature 466, 739-743 (2010)

13. Mashiko, H., Yamaguchi, T., Oguri, K., Suda, A. \& Gotoh, H. Characterizing inner-shell with spectral phase interferometry for direct electric-field reconstruction. Nature Commun. 5, 5599 (2014).

14. Kim, K. T. et al. Petahertz optical oscilloscope. Nature Photon. 7, 958-962 (2013)

15. Chini, M. et al. Sub-cycle oscillations in virtual states brought to light. Sci. Rep. 3, 1105 (2013)

16. Calegari, F. et al. Ultrafast electron dynamics in phenylalanine initiated by attosecond pulses. Science 346, 336-339 (2014).

17. Schultze, M. et al. Controlling dielectrics with the electric field of light. Nature 493, 75-78 (2013).

18. Schultze, M. et al. Attosecond band-gap dynamics in silicon. Science 346, 1348-1352 (2014).

19. Morkoc, H. et al. Large-band-gap SiC, III-V nitride, and II-VI ZnSe-based semiconductor device technologies. J. Appl. Phys. 76, 1363 (1994).

20. Tsaia, M.-H., Sankey, O. F., Schmidt, K. E. \& Tsong, I. S. T. Electronic structures of polar and nonpolar GaN surfaces. Mater. Sci. Eng. B 88, 40-46 (2002).

21. Strite, S. \& Morkoç, H. GaN, AlN, and InN: a review. J. Vac. Sci. Technol. B 10, 1237-1266 (1992).

22. Hofmann, M. et al. Noninstantaneous polarization dynamics in dielectric media. Optica 2, 151-157 (2015)

23. Zeller, J., Rudolph, W. \& Sheik-Bahae, M. Anomalous nonlinear photoresponse in a InGaN/GaN heterostructure. J. Appl. Phys. 95, 6152-6158 (2004).

24. Mashiko, H. et al. Double optical gating of high-order harmonic generation with carrier-envelope phase stabilized lasers. Phys. Rev. Lett. 100, 103906 (2008)

25. Ott, C. et al. Reconstruction and control of a time-dependent two-electron wave packet. Nature 516, 374-378 (2014)

26. Mücke, O. D., Tritschler, T., Wegener, M., Morgner, U. \& Kärtner, F. X. Carrier-wave Rabi flopping in GaAs using $5 \mathrm{fs}, 10^{12} \mathrm{~W} \mathrm{~cm} \mathrm{~cm}^{-2}$ pulses. Phys. Rev. Lett. 87, 057401 (2001). 
27. Tritschler, T., Mücke, O. D., Wegener, M., Morgner, U. \& Kärtner, F. X. Evidence for third-harmonic generation in disguise of second-harmonic generation in extreme nonlinear optics. Phys. Rev. Lett. 90, 217404 (2003).

28. Tritschler, T., Mücke, O. D. \& Wegener, M. Extreme nonlinear optics of two-level systems. Phys. Rev. A 68, 033404 (2003).

29. Mairesse, Y. \& Quéré, F. Frequency-resolved optical gating for complete reconstruction of attosecond bursts. Phys. Rev. A 71, 0011401(R) (2005).

30. Itatani, J. et al. Attosecond streak camera. Phys. Rev. Lett. 88, 173903 (2002).

\section{Acknowledgements}

We thank T. Tawara and K. Tateno of NTT Basic Research Laboratories and T. Ikeda of NTT Advance Technology Inc. for preparation of GaN material and fruitful discussions.

This work was supported by JSPS KAKENHI Grant No. 25706027.

\section{Author contributions}

H.M., K.O. and T.Y. performed the experiments and calculations. H.M., K.O., A.S. and H.G. planned and coordinated the project. H.M. and K.O. wrote the manuscript with contributions from all authors.

\section{Additional information}

Supplementary information is available in the online version of the paper. Reprints and permissions information is available online at www.nature.com/reprints. Correspondence and requests for materials should be addressed to H.M.

\section{Competing financial interests}

The authors declare no competing financial interests. 


\section{Methods}

Definition of deviation of optical density $(\Delta \mathrm{OD})$. The transient absorption spectrum at delay time $\tau$ between the IAP and NIR pulse is given by

$\Delta \mathrm{OD}(\omega, \tau)=\log \left[I_{\text {out }}(\omega, \tau) / I_{\mathrm{NIR}}(\omega, \tau)\right]$, where $\omega$ is the laser frequency. Here, $I_{\text {out }}(\omega, \tau)$ is the absorption spectrum of the IAP in the GaN without the NIR pulse, whereas $I_{\mathrm{NIR}}(\omega, \tau)$ is the absorption spectrum with the NIR pulse. Consequently, $\Delta \mathrm{OD}(\omega, \tau)$ monitors the spectral deviation in the presence of the NIR pulse.

Temporal characterization of IAP. The IAP is characterized using the attosecond streak method ${ }^{29,30}$ (for details of the IAP temporal characterization, see
Supplementary Section 2). In our experiment, the system configuration is similar to that used in the transient absorption spectroscopy. The collinearly propagating IAP and NIR pulse are focused into a gas jet of krypton atoms (50- $\mu \mathrm{m}$ interaction length; 740-mbar backing pressure). The estimated target intensity of the NIR pulse is approximately $5 \times 10^{10} \mathrm{~W} \mathrm{~cm}^{-2}$ in this

measurement. The ionized photoelectrons induced by the IAP are detected by a regular time-of-flight system. The temporal profile and phase of the IAP are reconstructed by the FROG-CRAB method ${ }^{29}$. Here, the IAP characterized with a centre photon energy $20.5 \mathrm{eV}$ has a duration of 660 as full-width at half-maximum. 